\title{
Some New Riemann-Liouville Fractional Integral Inequalities
}

\author{
Jessada Tariboon, ${ }^{1}$ Sotiris K. Ntouyas, ${ }^{2}$ and Weerawat Sudsutad ${ }^{1}$ \\ ${ }^{1}$ Department of Mathematics, Faculty of Applied Science, King Mongkut's University of Technology North Bangkok, Bangkok, Thailand \\ ${ }^{2}$ Department of Mathematics, University of Ioannina, 45110 Ioannina, Greece
}

Correspondence should be addressed to Jessada Tariboon; jessadat@kmutnb.ac.th

Received 12 December 2013; Accepted 23 January 2014; Published 10 March 2014

Academic Editor: Ram N. Mohapatra

Copyright (c) 2014 Jessada Tariboon et al. This is an open access article distributed under the Creative Commons Attribution License, which permits unrestricted use, distribution, and reproduction in any medium, provided the original work is properly cited.

In this paper, some new fractional integral inequalities are established.

\section{Introduction}

In [1] (see also [2]), the Grüss inequality is defined as the integral inequality that establishes a connection between the integral of the product of two functions and the product of the integrals. The inequality is as follows.

If $f$ and $g$ are two continuous functions on $[a, b]$ satisfying $m \leq f(t) \leq M$ and $p \leq g(t) \leq P$ for all $t \in[a, b]$, $m, M, p, P \in \mathbb{R}$, then

$$
\begin{aligned}
& \left|\frac{1}{b-a} \int_{a}^{b} f(t) g(t) d t-\frac{1}{(b-a)^{2}} \int_{a}^{b} f(t) d t \int_{a}^{b} g(t) d t\right| \\
& \quad \leq \frac{1}{4}(M-m)(P-p) .
\end{aligned}
$$

The literature on Grüss type inequalities is now vast, and many extensions of the classical inequality were intensively studied by many authors. In the past several years, by using the Riemann-Liouville fractional integrals, the fractional integral inequalities and applications have been addressed extensively by several researchers. For example, we refer the reader to [3-9] and the references cited therein. Dahmani et al. [10] gave the following fractional integral inequalities by using the Riemann-Liouville fractional integrals. Let $f$ and $g$ be two integrable functions on $[0, \infty)$ satisfying the following conditions:

$$
\begin{gathered}
m \leq f(t) \leq M, \quad p \leq g(t) \leq P \\
m, M, p, P \in \mathbb{R}, \quad t \in[0, \infty) .
\end{gathered}
$$

For all $t>0, \alpha>0, \beta>0$, then

$$
\begin{aligned}
& \left|\frac{t^{\alpha}}{\Gamma(\alpha+1)} J^{\alpha}(f g)(t)-J^{\alpha} f(t) J^{\alpha} g(t)\right| \\
& \leq\left(\frac{t^{\alpha}}{\Gamma(\alpha+1)}\right)^{2}(M-m)(P-p), \\
& \left(\frac{t^{\alpha}}{\Gamma(\alpha+1)} J^{\beta}(f g)(t)+\frac{t^{\beta}}{\Gamma(\beta+1)} J^{\alpha}(f g)(t)\right. \\
& \left.-J^{\alpha} f(t) J^{\beta} g(t)-J^{\beta} f(t) J^{\alpha} g(t)\right)^{2} \\
& \leq\left\{\left(M \frac{t^{\alpha}}{\Gamma(\alpha+1)}-J^{\alpha} f(t)\right)\left(J^{\beta} f(t)-m \frac{t^{\beta}}{\Gamma(\beta+1)}\right)\right. \\
& \quad+\left(J^{\alpha} f(t)-m \frac{t^{\alpha}}{\Gamma(\alpha+1)}\right) \\
& \left.\quad \times\left(M \frac{t^{\beta}}{\Gamma(\beta+1)}-J^{\beta} f(t)\right)\right\} \\
& \quad \times\left\{\left(P \frac{t^{\alpha}}{\Gamma(\alpha+1)}-J^{\alpha} g(t)\right)\left(J^{\beta} g(t)-p \frac{t^{\beta}}{\Gamma(\beta+1)}\right)\right. \\
& \quad+\left(J^{\alpha} g(t)-p \frac{t^{\alpha}}{\Gamma(\alpha+1)}\right) \\
& \left.\quad \times\left(P \frac{t^{\beta}}{\Gamma(\beta+1)}-J^{\beta} g(t)\right)\right\} .
\end{aligned}
$$


In this paper, we use the Riemann-Liouville fractional integrals to establish some new fractional integral inequalities of Grüss type. We replace the constants appeared as bounds of the functions $f$ and $g$, by four integrable functions. From our results, the above inequalities of [10] and the classical Grüss inequalities can be deduced as some special cases.

In Section 2 we briefly review the necessary definitions. Our results are given in Section 3. The proof technique is close to that presented in [10]. But the obtained results are new and also can be applied to unbounded functions as shown in examples.

\section{Preliminaries}

Definition 1. The Riemann-Liouville fractional integral of order $\alpha \geq 0$ of a function $g \in L^{1}((0, \infty), \mathbb{R})$ is defined by

$$
\begin{gathered}
J^{\alpha} f(t)=\int_{0}^{t} \frac{(t-s)^{\alpha-1}}{\Gamma(\alpha)} f(s) d s, \\
J^{0} f(t)=f(t),
\end{gathered}
$$

where $\Gamma$ is the gamma function.

For the convenience of establishing our results, we give the semigroup property:

$$
J^{\alpha} J^{\beta} f(t)=J^{\alpha+\beta} f(t), \quad \alpha \geq 0, \quad \beta \geq 0,
$$

which implies the commutative property

$$
J^{\alpha} J^{\beta} f(t)=J^{\beta} J^{\alpha} f(t) .
$$

From Definition 1, if $f(t)=t^{\gamma}$, then we have

$$
J^{\alpha} t^{\gamma}=\frac{\Gamma(\gamma+1)}{\Gamma(\alpha+\gamma+1)} t^{\alpha+\gamma}, \quad \alpha>0, \gamma>-1, t>0 .
$$

\section{Main Results}

Theorem 2. Let $f$ be an integrable function on $[0, \infty)$. Assume that

$\left(H_{1}\right)$ there exist two integrable functions $\varphi_{1}, \varphi_{2}$ on $[0, \infty)$ such that

$$
\varphi_{1}(t) \leq f(t) \leq \varphi_{2}(t) \quad \forall t \in[0, \infty) .
$$

Then, for $t>0, \alpha, \beta>0$, one has

$$
\begin{aligned}
& J^{\beta} \varphi_{1}(t) J^{\alpha} f(t)+J^{\alpha} \varphi_{2}(t) J^{\beta} f(t) \\
& \quad \geq J^{\alpha} \varphi_{2}(t) J^{\beta} \varphi_{1}(t)+J^{\alpha} f(t) J^{\beta} f(t) .
\end{aligned}
$$

Proof. From $\left(H_{1}\right)$, for all $\tau \geq 0, \rho \geq 0$, we have

$$
\left(\varphi_{2}(\tau)-f(\tau)\right)\left(f(\rho)-\varphi_{1}(\rho)\right) \geq 0 .
$$

Therefore

$$
\varphi_{2}(\tau) f(\rho)+\varphi_{1}(\rho) f(\tau) \geq \varphi_{1}(\rho) \varphi_{2}(\tau)+f(\tau) f(\rho) .
$$

Multiplying both sides of (11) by $(t-\tau)^{\alpha-1} / \Gamma(\alpha), \tau \in(0, t)$, we get

$$
\begin{aligned}
& f(\rho) \frac{(t-\tau)^{\alpha-1}}{\Gamma(\alpha)} \varphi_{2}(\tau)+\varphi_{1}(\rho) \frac{(t-\tau)^{\alpha-1}}{\Gamma(\alpha)} f(\tau) \\
& \quad \geq \varphi_{1}(\rho) \frac{(t-\tau)^{\alpha-1}}{\Gamma(\alpha)} \varphi_{2}(\tau)+f(\rho) \frac{(t-\tau)^{\alpha-1}}{\Gamma(\alpha)} f(\tau) .
\end{aligned}
$$

Integrating both sides of (12) with respect to $\tau$ on $(0, t)$, we obtain

$$
\begin{aligned}
& f(\rho) \int_{0}^{t} \frac{(t-\tau)^{\alpha-1}}{\Gamma(\alpha)} \varphi_{2}(\tau) d \tau \\
& \quad+\varphi_{1}(\rho) \int_{0}^{t} \frac{(t-\tau)^{\alpha-1}}{\Gamma(\alpha)} f(\tau) d \tau \\
& \geq \varphi_{1}(\rho) \int_{0}^{t} \frac{(t-\tau)^{\alpha-1}}{\Gamma(\alpha)} \varphi_{2}(\tau) d \tau \\
& \quad+f(\rho) \int_{0}^{t} \frac{(t-\tau)^{\alpha-1}}{\Gamma(\alpha)} f(\tau) d \tau,
\end{aligned}
$$

which yields

$$
\begin{aligned}
f(\rho) & J^{\alpha} \varphi_{2}(t)+\varphi_{1}(\rho) J^{\alpha} f(t) \\
& \geq \varphi_{1}(\rho) J^{\alpha} \varphi_{2}(t)+f(\rho) J^{\alpha} f(t) .
\end{aligned}
$$

Multiplying both sides of (14) by $(t-\rho)^{\beta-1} / \Gamma(\beta), \rho \in(0, t)$, we have

$$
\begin{aligned}
J^{\alpha} \varphi_{2}(t) & \frac{(t-\rho)^{\beta-1}}{\Gamma(\beta)} f(\rho)+J^{\alpha} f(t) \frac{(t-\rho)^{\beta-1}}{\Gamma(\beta)} \varphi_{1}(\rho) \\
\geq & J^{\alpha} \varphi_{2}(t) \frac{(t-\rho)^{\beta-1}}{\Gamma(\beta)} \varphi_{1}(\rho)+J^{\alpha} f(t) \frac{(t-\rho)^{\beta-1}}{\Gamma(\beta)} f(\rho) .
\end{aligned}
$$

Integrating both sides of (15) with respect to $\rho$ on $(0, t)$, we get

$$
\begin{aligned}
J^{\alpha} \varphi_{2}(t) \int_{0}^{t} \frac{(t-\rho)^{\beta-1}}{\Gamma(\beta)} f(\rho) d \rho \\
+J^{\alpha} f(t) \int_{0}^{t} \frac{(t-\rho)^{\beta-1}}{\Gamma(\beta)} \varphi_{1}(\rho) d \rho \\
\geq J^{\alpha} \varphi_{2}(t) \int_{0}^{t} \frac{(t-\rho)^{\beta-1}}{\Gamma(\beta)} \varphi_{1}(\rho) d \rho \\
+J^{\alpha} f(t) \int_{0}^{t} \frac{(t-\rho)^{\beta-1}}{\Gamma(\beta)} f(\rho) d \rho .
\end{aligned}
$$

Hence, we deduce inequality (9) as requested. This completes the proof. 
As a special case of Theorem 2, we obtain the following result.

Corollary 3. Let $f$ be an integrable function on $[0, \infty)$ satisfying $m \leq f(t) \leq M$, for all $t \in[0, \infty)$ and $m, M \in \mathbb{R}$. Then, for $t>0$ and $\alpha, \beta>0$, one has

$$
\begin{aligned}
& m \frac{t^{\beta}}{\Gamma(\beta+1)} J^{\alpha} f(t)+M \frac{t^{\alpha}}{\Gamma(\alpha+1)} J^{\beta} f(t) \\
& \quad \geq m M \frac{t^{\alpha+\beta}}{\Gamma(\alpha+1) \Gamma(\beta+1)}+J^{\alpha} f(t) J^{\beta} f(t) .
\end{aligned}
$$

Example 4. Let $f$ be a function satisfying $t \leq f(t) \leq t+1$ for $t \in[0, \infty)$. Then, for $t>0$ and $\alpha>0$, we have

$$
\begin{aligned}
& \left(\frac{2 t^{\alpha+1}}{\Gamma(\alpha+2)}+\frac{t^{\alpha}}{\Gamma(\alpha+1)}\right) J^{\alpha} f(t) \\
& \quad \geq\left(\frac{t^{\alpha+1}}{\Gamma(\alpha+2)}+\frac{t^{\alpha}}{\Gamma(\alpha+1)}\right)\left(\frac{t^{\alpha+1}}{\Gamma(\alpha+2)}\right)+\left(J^{\alpha} f(t)\right)^{2} .
\end{aligned}
$$

Theorem 5. Let $f$ and $g$ be two integrable functions on $[0, \infty)$. Suppose that $\left(H_{1}\right)$ holds and moreover one assumes that

$\left(H_{2}\right)$ there exist $\psi_{1}$ and $\psi_{2}$ integrable functions on $[0, \infty)$ such that

$$
\psi_{1}(t) \leq g(t) \leq \psi_{2}(t), \quad \forall t \in[0, \infty)
$$

Then, for $t>0, \alpha, \beta>0$, the following inequalities hold:

(a) $J^{\beta} \psi_{1}(t) J^{\alpha} f(t)+J^{\alpha} \varphi_{2}(t) J^{\beta} g(t)$

$$
\geq J^{\beta} \psi_{1}(t) J^{\alpha} \varphi_{2}(t)+J^{\alpha} f(t) J^{\beta} g(t),
$$

(b) $J^{\beta} \varphi_{1}(t) J^{\alpha} g(t)+J^{\alpha} \psi_{2}(t) J^{\beta} f(t)$

$$
\geq J^{\beta} \varphi_{1}(t) J^{\alpha} \psi_{2}(t)+J^{\beta} f(t) J^{\alpha} g(t),
$$

(c) $J^{\alpha} \varphi_{2}(t) J^{\beta} \psi_{2}(t)+J^{\alpha} f(t) J^{\beta} g(t)$

$$
\geq J^{\alpha} \varphi_{2}(t) J^{\beta} g(t)+J^{\beta} \psi_{2}(t) J^{\alpha} f(t),
$$

(d) $J^{\alpha} \varphi_{1}(t) J^{\beta} \psi_{1}(t)+J^{\alpha} f(t) J^{\beta} g(t)$

$$
\geq J^{\alpha} \varphi_{1}(t) J^{\beta} g(t)+J^{\beta} \psi_{1}(t) J^{\alpha} f(t) .
$$

Proof. To prove $(a)$, from $\left(H_{1}\right)$ and $\left(H_{2}\right)$, we have for $t \in$ $[0, \infty)$ that

$$
\left(\varphi_{2}(\tau)-f(\tau)\right)\left(g(\rho)-\psi_{1}(\rho)\right) \geq 0
$$

Therefore

$$
\varphi_{2}(\tau) g(\rho)+\psi_{1}(\rho) f(\tau) \geq \psi_{1}(\rho) \varphi_{2}(\tau)+f(\tau) g(\rho) \text {. }
$$

Multiplying both sides of (22) by $(t-\tau)^{\alpha-1} / \Gamma(\alpha), \tau \in(0, t)$, we get

$$
\begin{aligned}
& g(\rho) \frac{(t-\tau)^{\alpha-1}}{\Gamma(\alpha)} \varphi_{2}(\tau)+\psi_{1}(\rho) \frac{(t-\tau)^{\alpha-1}}{\Gamma(\alpha)} f(\tau) \\
& \quad \geq \psi_{1}(\rho) \frac{(t-\tau)^{\alpha-1}}{\Gamma(\alpha)} \varphi_{2}(\tau)+g(\rho) \frac{(t-\tau)^{\alpha-1}}{\Gamma(\alpha)} f(\tau) .
\end{aligned}
$$

Integrating both sides of (23) with respect to $\tau$ on $(0, t)$, we obtain

$$
\begin{aligned}
& g(\rho) \int_{0}^{t} \frac{(t-\tau)^{\alpha-1}}{\Gamma(\alpha)} \varphi_{2}(\tau) d \tau \\
& \quad+\psi_{1}(\rho) \int_{0}^{t} \frac{(t-\tau)^{\alpha-1}}{\Gamma(\alpha)} f(\tau) d \tau \\
& \geq \psi_{1}(\rho) \int_{0}^{t} \frac{(t-\tau)^{\alpha-1}}{\Gamma(\alpha)} \varphi_{2}(\tau) d \tau \\
& \quad+g(\rho) \int_{0}^{t} \frac{(t-\tau)^{\alpha-1}}{\Gamma(\alpha)} f(\tau) d \tau .
\end{aligned}
$$

Then we have

$$
\begin{aligned}
& g(\rho) J^{\alpha} \varphi_{2}(t)+\psi_{1}(\rho) J^{\alpha} f(t) \\
& \quad \geq \psi_{1}(\rho) J^{\alpha} \varphi_{2}(t)+g(\rho) J^{\alpha} f(t) .
\end{aligned}
$$

Multiplying both sides of (25) by $(t-\rho)^{\beta-1} / \Gamma(\beta), \rho \in(0, t)$, we have

$$
\begin{aligned}
J^{\alpha} \varphi_{2}(t) & \frac{(t-\rho)^{\beta-1}}{\Gamma(\beta)} g(\rho)+J^{\alpha} f(t) \frac{(t-\rho)^{\beta-1}}{\Gamma(\beta)} \psi_{1}(\rho) \\
& \geq J^{\alpha} \varphi_{2}(t) \frac{(t-\rho)^{\beta-1}}{\Gamma(\beta)} \psi_{1}(\rho)+J^{\alpha} f(t) \frac{(t-\rho)^{\beta-1}}{\Gamma(\beta)} g(\rho) .
\end{aligned}
$$

Integrating both sides of (26) with respect to $\rho$ on $(0, t)$, we get the desired inequality $(a)$.

To prove $(b)-(d)$, we use the following inequalities:

$$
\begin{aligned}
& \text { (b) }\left(\psi_{2}(\tau)-g(\tau)\right)\left(f(\rho)-\varphi_{1}(\rho)\right) \geq 0, \\
& \text { (c) }\left(\varphi_{2}(\tau)-f(\tau)\right)\left(g(\rho)-\psi_{2}(\rho)\right) \leq 0, \\
& \text { (d) }\left(\varphi_{1}(\tau)-f(\tau)\right)\left(g(\rho)-\psi_{1}(\rho)\right) \leq 0 .
\end{aligned}
$$

As a special case of Theorem 5, we have the following corollary.

Corollary 6. Let $f$ and $g$ be two integrable functions on $[0, \infty)$. Assume that

$\left(H_{3}\right)$ there exist real constants $m, M, n, N$ such that

$$
m \leq f(t) \leq M, \quad n \leq g(t) \leq N \quad \forall t \in[0, \infty) .
$$


Then, fort $>0, \alpha, \beta>0$, we have

$$
\begin{gathered}
\left(a_{1}\right) \frac{n t^{\beta}}{\Gamma(\beta+1)} J^{\alpha} f(t)+\frac{M t^{\alpha}}{\Gamma(\alpha+1)} J^{\beta} g(t) \\
\geq \frac{n M t^{\alpha+\beta}}{\Gamma(\alpha+1) \Gamma(\beta+1)}+J^{\alpha} f(t) J^{\beta} g(t), \\
\left(b_{1}\right) \frac{m t^{\beta}}{\Gamma(\beta+1)} J^{\alpha} g(t)+\frac{N t^{\alpha}}{\Gamma(\alpha+1)} J^{\beta} f(t) \\
\geq \frac{m N t^{\alpha+\beta}}{\Gamma(\alpha+1) \Gamma(\beta+1)}+J^{\beta} f(t) J^{\alpha} g(t), \\
\left(c_{1}\right) \frac{M N t^{\alpha+\beta}}{\Gamma(\alpha+1) \Gamma(\beta+1)}+J^{\alpha} f(t) J^{\beta} g(t) \\
\geq \frac{M t^{\alpha}}{\Gamma(\alpha+1)} J^{\beta} g(t)+\frac{N t^{\beta}}{\Gamma(\beta+1)} J^{\alpha} f(t), \\
\left(d_{1}\right) \frac{m n t^{\alpha+\beta}}{\Gamma(\alpha+1) \Gamma(\beta+1)}+J^{\alpha} f(t) J^{\beta} g(t) \\
\geq \frac{m t^{\alpha}}{\Gamma(\alpha+1)} J^{\beta} g(t)+\frac{n t^{\beta}}{\Gamma(\beta+1)} J^{\alpha} f(t) .
\end{gathered}
$$

Lemma 7. Let $f$ be an integrable function on $[0, \infty)$ and let $\varphi_{1}, \varphi_{2}$ be two integrable functions on $[0, \infty)$. Assume that the condition $\left(H_{1}\right)$ holds. Then, for $t>0, \alpha>0$, we have

$$
\begin{aligned}
& \frac{t^{\alpha}}{\Gamma(\alpha+1)} J^{\alpha} f^{2}(t)-\left(J^{\alpha} f(t)\right)^{2} \\
& =\left(J^{\alpha} \varphi_{2}(t)-J^{\alpha} f(t)\right)\left(J^{\alpha} f(t)-J^{\alpha} \varphi_{1}(t)\right) \\
& \quad-\frac{t^{\alpha}}{\Gamma(\alpha+1)} J^{\alpha}\left(\left(\varphi_{2}(t)-f(t)\right)\left(f(t)-\varphi_{1}(t)\right)\right) \\
& \quad+\frac{t^{\alpha}}{\Gamma(\alpha+1)} J^{\alpha} \varphi_{1} f(t)-J^{\alpha} \varphi_{1}(t) J^{\alpha} f(t) \\
& \quad+\frac{t^{\alpha}}{\Gamma(\alpha+1)} J^{\alpha} \varphi_{2} f(t)-J^{\alpha} \varphi_{2}(t) J^{\alpha} f(t) \\
& \quad+J^{\alpha} \varphi_{1}(t) J^{\alpha} \varphi_{2}(t)-\frac{t^{\alpha}}{\Gamma(\alpha+1)} J^{\alpha} \varphi_{1} \varphi_{2}(t) .
\end{aligned}
$$

Proof. For any $\tau>0$ and $\rho>0$, we have

$$
\begin{aligned}
\left(\varphi_{2}(\rho)-f(\rho)\right)\left(f(\tau)-\varphi_{1}(\tau)\right) \\
\quad+\left(\varphi_{2}(\tau)-f(\tau)\right)\left(f(\rho)-\varphi_{1}(\rho)\right) \\
\quad-\left(\varphi_{2}(\tau)-f(\tau)\right)\left(f(\tau)-\varphi_{1}(\tau)\right) \\
\quad-\left(\varphi_{2}(\rho)-f(\rho)\right)\left(f(\rho)-\varphi_{1}(\rho)\right) \\
=f^{2}(\tau)+f^{2}(\rho)-2 f(\tau) f(\rho) \\
\quad+\varphi_{2}(\rho) f(\tau)+\varphi_{1}(\tau) f(\rho)-\varphi_{1}(\tau) \varphi_{2}(\rho) \\
\quad+\varphi_{2}(\tau) f(\rho)+\varphi_{1}(\rho) f(\tau)-\varphi_{1}(\rho) \varphi_{2}(\tau) \\
\quad-\varphi_{2}(\tau) f(\tau)+\varphi_{1}(\tau) \varphi_{2}(\tau)-\varphi_{1}(\tau) f(\tau) \\
\quad-\varphi_{2}(\rho) f(\rho)+\varphi_{1}(\rho) \varphi_{2}(\rho)-\varphi_{1}(\rho) f(\rho) .
\end{aligned}
$$

Multiplying (31) by $(t-\tau)^{\alpha-1} / \Gamma(\alpha), \tau \in(0, t), t>0$ and integrating the resulting identity with respect to $\tau$, from 0 to $t$, we get

$$
\begin{aligned}
\left(\varphi_{2}(\rho)\right. & -f(\rho))\left(J^{\alpha} f(t)-J^{\alpha} \varphi_{1}(t)\right) \\
& +\left(J^{\alpha} \varphi_{2}(t)-J^{\alpha} f(t)\right)\left(f(\rho)-\varphi_{1}(\rho)\right) \\
& -J^{\alpha}\left(\left(\varphi_{2}(t)-f(t)\right)\left(f(t)-\varphi_{1}(t)\right)\right) \\
& -\left(\varphi_{2}(\rho)-f(\rho)\right)\left(f(\rho)-\varphi_{1}(\rho)\right) \frac{t^{\alpha}}{\Gamma(\alpha+1)} \\
= & J^{\alpha} f^{2}(t)+f^{2}(\rho) \frac{t^{\alpha}}{\Gamma(\alpha+1)}-2 f(\rho) J^{\alpha} f(t) \\
& +\varphi_{2}(\rho) J^{\alpha} f(t)+f(\rho) J^{\alpha} \varphi_{1}(t) \\
& -\varphi_{2}(\rho) J^{\alpha} \varphi_{1}(t)+f(\rho) J^{\alpha} \varphi_{2}(t) \\
& +\varphi_{1}(\rho) J^{\alpha} f(t)-\varphi_{1}(\rho) J^{\alpha} \varphi_{2}(t) \\
& -J^{\alpha} \varphi_{2} f(t)+J^{\alpha} \varphi_{1} \varphi_{2}(t)-J^{\alpha} \varphi_{1} f(t) \\
& -\varphi_{2}(\rho) f(\rho) \frac{t^{\alpha}}{\Gamma(\alpha+1)}+\varphi_{1}(\rho) \varphi_{2}(\rho) \frac{t^{\alpha}}{\Gamma(\alpha+1)} \\
& -\varphi_{1}(\rho) f(\rho) \frac{t^{\alpha}}{\Gamma(\alpha+1)} .
\end{aligned}
$$

Multiplying (32) by $(t-\rho)^{\alpha-1} / \Gamma(\alpha), \rho \in(0, t), t>0$ and integrating the resulting identity with respect to $\rho$, from 0 to $t$, we have

$$
\begin{aligned}
\left(J^{\alpha} \varphi_{2}\right. & \left.(t)-J^{\alpha} f(t)\right)\left(J^{\alpha} f(t)-J^{\alpha} \varphi_{1}(t)\right) \\
& +\left(J^{\alpha} \varphi_{2}(t)-J^{\alpha} f(t)\right)\left(J^{\alpha} f(t)-J^{\alpha} \varphi_{1}(t)\right) \\
& -J^{\alpha}\left(\left(\varphi_{2}(t)-f(t)\right)\left(f(t)-\varphi_{1}(t)\right)\right) \frac{t^{\alpha}}{\Gamma(\alpha+1)} \\
& -J^{\alpha}\left(\left(\varphi_{2}(t)-f(t)\right)\left(f(t)-\varphi_{1}(t)\right)\right) \frac{t^{\alpha}}{\Gamma(\alpha+1)} \\
= & \frac{t^{\alpha}}{\Gamma(\alpha+1)} J^{\alpha} f^{2}(t)+\frac{t^{\alpha}}{\Gamma(\alpha+1)} J^{\alpha} f^{2}(t) \\
& -2 J^{\alpha} f(t) J^{\alpha} f(t)+J^{\alpha} \varphi_{2}(t) J^{\alpha} f(t) \\
& +J^{\alpha} \varphi_{1}(t) J^{\alpha} f(t)-J^{\alpha} \varphi_{1}(t) J^{\alpha} \varphi_{2}(t) \\
& +J^{\alpha} \varphi_{2}(t) J^{\alpha} f(t)+J^{\alpha} \varphi_{1}(t) J^{\alpha} f(t) \\
& -J^{\alpha} \varphi_{1}(t) J^{\alpha} \varphi_{2}(t)-\frac{t^{\alpha}}{\Gamma(\alpha+1)} J^{\alpha} \varphi_{2} f(t) \\
& +\frac{t^{\alpha}}{\Gamma(\alpha+1)} J^{\alpha} \varphi_{1} \varphi_{2}(t)-\frac{t^{\alpha}}{\Gamma(\alpha+1)} J^{\alpha} \varphi_{1} f(t) \\
& -\frac{t^{\alpha}}{\Gamma(\alpha+1)} J^{\alpha} \varphi_{2} f(t)+\frac{t^{\alpha}}{\Gamma(\alpha+1)} J^{\alpha} \varphi_{1} \varphi_{2}(t) \\
& -\frac{t^{\alpha}}{\Gamma(\alpha+1)} J^{\alpha} \varphi_{1} f(t),
\end{aligned}
$$

which implies (30). 
If $\varphi_{1}(t) \equiv m$ and $\varphi_{2}(t) \equiv M, m, M \in \mathbb{R}$, for all $t \in[0, \infty)$, then inequality (30) reduces to the following corollary $[10$, Lemma 3.2].

Corollary 8. Let $f$ be an integrable function on $[0, \infty)$ satisfying $m \leq f(t) \leq M$, for all $t \in[0, \infty)$. Then, for all $t>0$, $\alpha>0$, one has

$$
\begin{aligned}
& \frac{t^{\alpha}}{\Gamma(\alpha+1)} J^{\alpha} f^{2}(t)-\left(J^{\alpha} f(t)\right)^{2} \\
& =\left(M \frac{t^{\alpha}}{\Gamma(\alpha+1)}-J^{\alpha} f(t)\right)\left(J^{\alpha} f(t)-m \frac{t^{\alpha}}{\Gamma(\alpha+1)}\right) \\
& \quad-\frac{t^{\alpha}}{\Gamma(\alpha+1)} J^{\alpha}((M-f(t))(f(t)-m)) .
\end{aligned}
$$

Theorem 9. Let $f$ and $g$ be two integrable functions on $[0, \infty)$ and let $\varphi_{1}, \varphi_{2}, \psi_{1}$, and $\psi_{2}$ be four integrable functions on $[0, \infty)$ satisfying the conditions $\left(H_{1}\right)$ and $\left(H_{2}\right)$ on $[0, \infty)$. Then, for all $t>0, \alpha>0$, one has

$$
\begin{gathered}
\left|\frac{t^{\alpha}}{\Gamma(\alpha+1)} J^{\alpha} f g(t)-J^{\alpha} f(t) J^{\alpha} g(t)\right| \\
\leq \sqrt{T\left(f, \varphi_{1}, \varphi_{2}\right) T\left(g, \psi_{1}, \psi_{2}\right)},
\end{gathered}
$$

where $T(u, v, w)$ is defined by

$$
\begin{aligned}
T(u, v, w)= & \left(J^{\alpha} w(t)-J^{\alpha} u(t)\right)\left(J^{\alpha} u(t)-J^{\alpha} v(t)\right) \\
& +\frac{t^{\alpha}}{\Gamma(\alpha+1)} J^{\alpha} v u(t)-J^{\alpha} v(t) J^{\alpha} u(t) \\
& +\frac{t^{\alpha}}{\Gamma(\alpha+1)} J^{\alpha} w u(t)-J^{\alpha} w(t) J^{\alpha} u(t) \\
& +J^{\alpha} v(t) J^{\alpha} w(t)-\frac{t^{\alpha}}{\Gamma(\alpha+1)} J^{\alpha} v w(t) .
\end{aligned}
$$

Proof. Let $f$ and $g$ be two integrable functions defined on $[0, \infty)$ satisfying $\left(H_{1}\right)$ and $\left(H_{2}\right)$. Define

$$
\begin{array}{r}
H(\tau, \rho):=(f(\tau)-f(\rho))(g(\tau)-g(\rho)), \\
\tau, \rho \in(0, t), t>0 .
\end{array}
$$

Multiplying both sides of (37) by $(t-\tau)^{\alpha-1}(t-\rho)^{\alpha-1} / \Gamma^{2}(\alpha)$, $\tau, \rho \in(0, t)$ and integrating the resulting identity with respect to $\tau$ and $\rho$, from 0 to $t$, we can state that

$$
\begin{gathered}
\frac{1}{2 \Gamma^{2}(\alpha)} \iint_{0}^{t}(t-\tau)^{\alpha-1}(t-\rho)^{\alpha-1} H(\tau, \rho) d \tau d \rho \\
=\frac{t^{\alpha}}{\Gamma(\alpha+1)} J^{\alpha} f g(t)-J^{\alpha} f(t) J^{\alpha} g(t) .
\end{gathered}
$$

Applying the Cauchy-Schwarz inequality to (38), we have

$$
\begin{aligned}
& \left(\frac{t^{\alpha}}{\Gamma(\alpha+1)} J^{\alpha} f g(t)-J^{\alpha} f(t) J^{\alpha} g(t)\right)^{2} \\
& \quad \leq\left(\frac{t^{\alpha}}{\Gamma(\alpha+1)} J^{\alpha} f^{2}(t)-\left(J^{\alpha} f(t)\right)^{2}\right) \\
& \quad \times\left(\frac{t^{\alpha}}{\Gamma(\alpha+1)} J^{\alpha} g^{2}(t)-\left(J^{\alpha} g(t)\right)^{2}\right) .
\end{aligned}
$$

Since $\left(\varphi_{2}(t)-f(t)\right)\left(f(t)-\varphi_{1}(t)\right) \geq 0$ and $\left(\psi_{2}(t)-g(t)\right)(g(t)-$ $\left.\psi_{1}(t)\right) \geq 0$, for $t \in[0, \infty)$, we have

$$
\begin{aligned}
& \frac{t^{\alpha}}{\Gamma(\alpha+1)} J^{\alpha}\left(\left(\varphi_{2}(t)-f(t)\right)\left(f(t)-\varphi_{1}(t)\right)\right) \geq 0, \\
& \frac{t^{\alpha}}{\Gamma(\alpha+1)} J^{\alpha}\left(\left(\psi_{2}(t)-g(t)\right)\left(g(t)-\psi_{1}(t)\right)\right) \geq 0 .
\end{aligned}
$$

Thus, from Lemma 7, we get

$$
\begin{aligned}
& \frac{t^{\alpha}}{\Gamma(\alpha+1)} J^{\alpha} f^{2}(t)-\left(J^{\alpha} f(t)\right)^{2} \\
& \leq\left(J^{\alpha} \varphi_{2}(t)-J^{\alpha} f(t)\right)\left(J^{\alpha} f(t)-J^{\alpha} \varphi_{1}(t)\right) \\
&+\frac{t^{\alpha}}{\Gamma(\alpha+1)} J^{\alpha} \varphi_{1} f(t)-J^{\alpha} \varphi_{1}(t) J^{\alpha} f(t) \\
&+\frac{t^{\alpha}}{\Gamma(\alpha+1)} J^{\alpha} \varphi_{2} f(t)-J^{\alpha} \varphi_{2}(t) J^{\alpha} f(t) \\
&+J^{\alpha} \varphi_{1}(t) J^{\alpha} \varphi_{2}(t)-\frac{t^{\alpha}}{\Gamma(\alpha+1)} J^{\alpha} \varphi_{1} \varphi_{2}(t) \\
&= T\left(f, \varphi_{1}, \varphi_{2}\right), \\
& \frac{t^{\alpha}}{\Gamma(\alpha+} J^{\alpha} g^{2}(t)-\left(J^{\alpha} g(t)\right)^{2} \\
& \leq\left(J^{\alpha} \psi_{2}(t)-J^{\alpha} g(t)\right)\left(J^{\alpha} g(t)-J^{\alpha} \psi_{1}(t)\right) \\
&+\frac{t^{\alpha}}{\Gamma(\alpha+1)} J^{\alpha} \psi_{1} g(t)-J^{\alpha} \psi_{1}(t) J^{\alpha} g(t) \\
&+\frac{t^{\alpha}}{\Gamma(\alpha+1)} J^{\alpha} \psi_{2} g(t)-J^{\alpha} \psi_{2}(t) J^{\alpha} g(t) \\
&+J^{\alpha} \psi_{1}(t) J^{\alpha} \psi_{2}(t)-\frac{t^{\alpha}}{\Gamma(\alpha+1)} J^{\alpha} \psi_{1} \psi_{2}(t) \\
&= T\left(g, \psi_{1}, \psi_{2}\right) .
\end{aligned}
$$

From (39), (41), and (42), we obtain (35).

Remark 10. If $T\left(f, \varphi_{1}, \varphi_{2}\right)=T(f, m, M)$ and $T\left(g, \psi_{1}, \psi_{2}\right)=$ $T(g, p, P), m, M, p, P \in \mathbb{R}$, then inequality (35) reduces to

$$
\begin{aligned}
& \left|\frac{t^{\alpha}}{\Gamma(\alpha+1)} J^{\alpha} f g(t)-J^{\alpha} f(t) J^{\alpha} g(t)\right| \\
& \quad \leq\left(\frac{t^{\alpha}}{2 \Gamma(\alpha+1)}\right)^{2}(M-m)(P-p) .
\end{aligned}
$$

See [10, Theorem 3.1]. 
Example 11. Let $f$ and $g$ be two functions satisfying $t \leq$ $f(t) \leq t+1$ and $t-1 \leq g(t) \leq t$ for $t \in[0, \infty)$. Then, for $t>0$ and $\alpha>0$, we have

$$
\begin{array}{r}
\left|\frac{t^{\alpha}}{\Gamma(\alpha+1)} J^{\alpha} f g(t)-J^{\alpha} f(t) J^{\alpha} g(t)\right| \\
\quad \leq \sqrt{T(f, t, t+1) T(g, t-1, t)},
\end{array}
$$

where

$$
\begin{aligned}
& T(f, t, t+1) \\
& =\left(\frac{t^{\alpha+1}}{\Gamma(\alpha+2)}+\frac{t^{\alpha}}{\Gamma(\alpha+1)}-J^{\alpha} f(t)\right) \\
& \times\left(J^{\alpha} f(t)-\frac{t^{\alpha+1}}{\Gamma(\alpha+2)}\right) \\
& +\frac{t^{\alpha}}{\Gamma(\alpha+1)} J^{\alpha}(t f)(t)-\frac{t^{\alpha+1}}{\Gamma(\alpha+2)} J^{\alpha} f(t) \\
& +\frac{t^{\alpha}}{\Gamma(\alpha+1)} J^{\alpha}((t+1) f)(t) \\
& -\left(\frac{t^{\alpha+1}}{\Gamma(\alpha+2)}+\frac{t^{\alpha}}{\Gamma(\alpha+1)}\right) J^{\alpha} f(t) \\
& +\left(\frac{t^{\alpha+1}}{\Gamma(\alpha+2)}\right)\left(\frac{t^{\alpha+1}}{\Gamma(\alpha+2)}+\frac{t^{\alpha}}{\Gamma(\alpha+1)}\right) \\
& -\frac{t^{\alpha}}{\Gamma(\alpha+1)}\left(\frac{2 t^{\alpha+2}}{\Gamma(\alpha+3)}+\frac{t^{\alpha+1}}{\Gamma(\alpha+2)}\right), \\
& T(g, t-1, t) \\
& =\left(\frac{t^{\alpha+1}}{\Gamma(\alpha+2)}-J^{\alpha} g(t)\right) \\
& \times\left(J^{\alpha} g(t)-\frac{t^{\alpha+1}}{\Gamma(\alpha+2)}+\frac{t^{\alpha}}{\Gamma(\alpha+1)}\right) \\
& +\frac{t^{\alpha}}{\Gamma(\alpha+1)} J^{\alpha}((t-1) g)(t) \\
& -\left(\frac{t^{\alpha+1}}{\Gamma(\alpha+2)}-\frac{t^{\alpha}}{\Gamma(\alpha+1)}\right) J^{\alpha} g(t) \\
& +\frac{t^{\alpha}}{\Gamma(\alpha+1)} J^{\alpha}(\operatorname{tg})(t)-\left(\frac{t^{\alpha+1}}{\Gamma(\alpha+2)}\right) J^{\alpha} g(t) \\
& +\left(\frac{t^{\alpha+1}}{\Gamma(\alpha+2)}-\frac{t^{\alpha}}{\Gamma(\alpha+1)}\right)\left(\frac{t^{\alpha+1}}{\Gamma(\alpha+2)}\right) \\
& -\frac{t^{\alpha}}{\Gamma(\alpha+1)}\left(\frac{2 t^{\alpha+2}}{\Gamma(\alpha+3)}-\frac{t^{\alpha+1}}{\Gamma(\alpha+2)}\right) \text {. }
\end{aligned}
$$

\section{Conflict of Interests}

The authors declare that there is no conflict of interests regarding the publication of this paper.

\section{Acknowledgments}

This research was funded by King Mongkut's University of Technology North Bangkok, Thailand. Project code: KMUTNB-GRAD-56-02. Sotiris K. Ntouyas is a member of Nonlinear Analysis and Applied Mathematics (NAAM)Research Group at King Abdulaziz University, Jeddah, Saudi Arabia.

\section{References}

[1] G. Grüss, "Über das Maximum des absoluten Betrages von $1 /(b-a) \int_{a}^{b} f(t) g(t) d t-\left(1 /(b-a)^{2}\right) \int_{a}^{b} f(t) d t \int_{a}^{b} g(t) d t$," Mathematische Zeitschrift, vol. 39, no. 1, pp. 215-226, 1935.

[2] D. S. Mitrinović, J. E. Pečarić, and A. M. Fink, Classical and New Inequalities in Analysis, vol. 61 of Mathematics and Its Applications, Kluwer Academic, Dordrecht, The Netherlands, 1993.

[3] G. A. Anastassiou, "Opial type inequalities involving fractional derivatives of two functions and applications," Computers \& Mathematics with Applications, vol. 48, no. 10-11, pp. 1701-1731, 2004.

[4] Z. Denton and A. S. Vatsala, "Fractional integral inequalities and applications," Computers \& Mathematics with Applications, vol. 59, no. 3, pp. 1087-1094, 2010.

[5] S. Belarbi and Z. Dahmani, "On some new fractional integral inequalities," Journal of Inequalities in Pure and Applied Mathematics, vol. 10, no. 3, article 86, 2009.

[6] Z. Dahmani, "New inequalities in fractional integrals," International Journal of Nonlinear Science, vol. 9, no. 4, pp. 493-497, 2010.

[7] W. T. Sulaiman, "Some new fractional integral inequalities," Journal of Mathematical Analysis, vol. 2, no. 2, pp. 23-28, 2011.

[8] M. Z. Sarikaya and H. Ogunmez, "On new inequalities via Riemann-Liouville fractional integration," Abstract and Applied Analysis, vol. 2012, Article ID 428983, 10 pages, 2012.

[9] C. Zhu, W. Yang, and Q. Zhao, "Some new fractional $q$ integral Grüss-type inequalities and other inequalities," Journal of Inequalities and Applications, vol. 2012, article 299, 15 pages, 2012.

[10] Z. Dahmani, L. Tabharit, and S. Taf, "New generalisations of Gruss inequality using Riemann-Liouville fractional integrals," Bulletin of Mathematical Analysis and Applications, vol. 2, no. 3, pp. 93-99, 2010. 


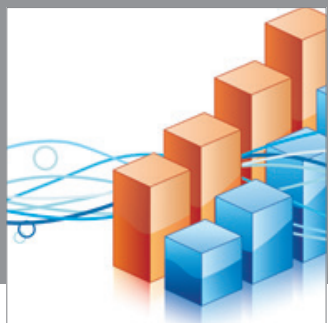

Advances in

Operations Research

mansans

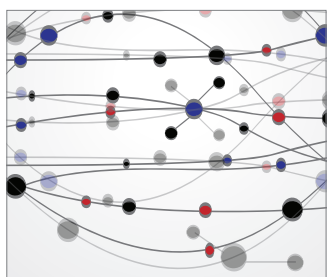

The Scientific World Journal
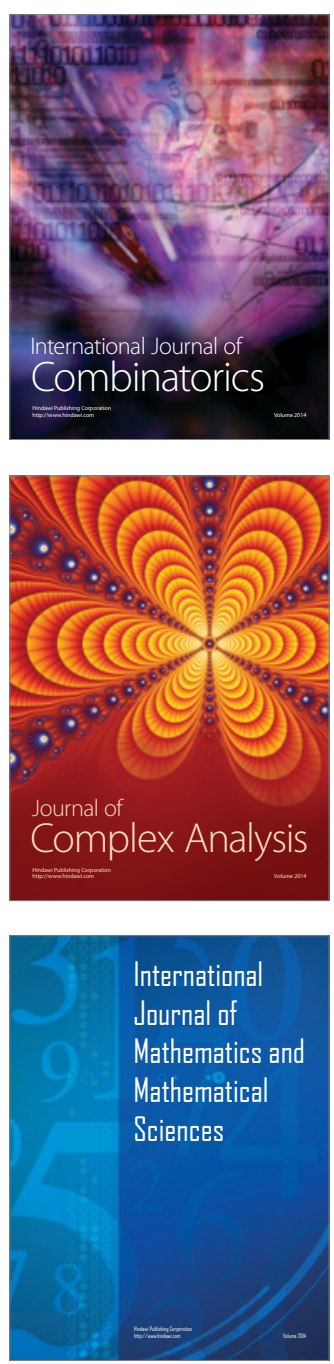
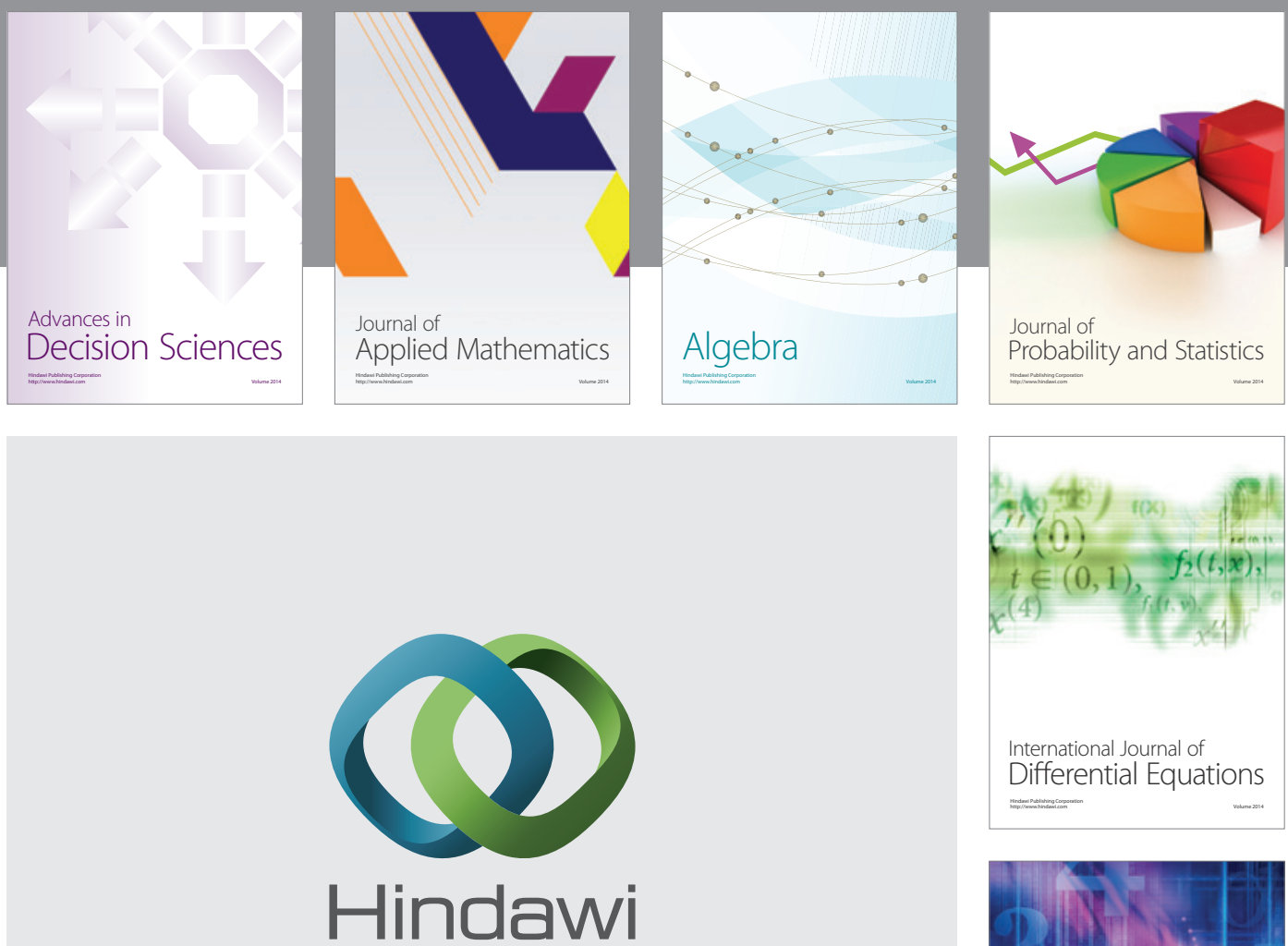

Submit your manuscripts at http://www.hindawi.com
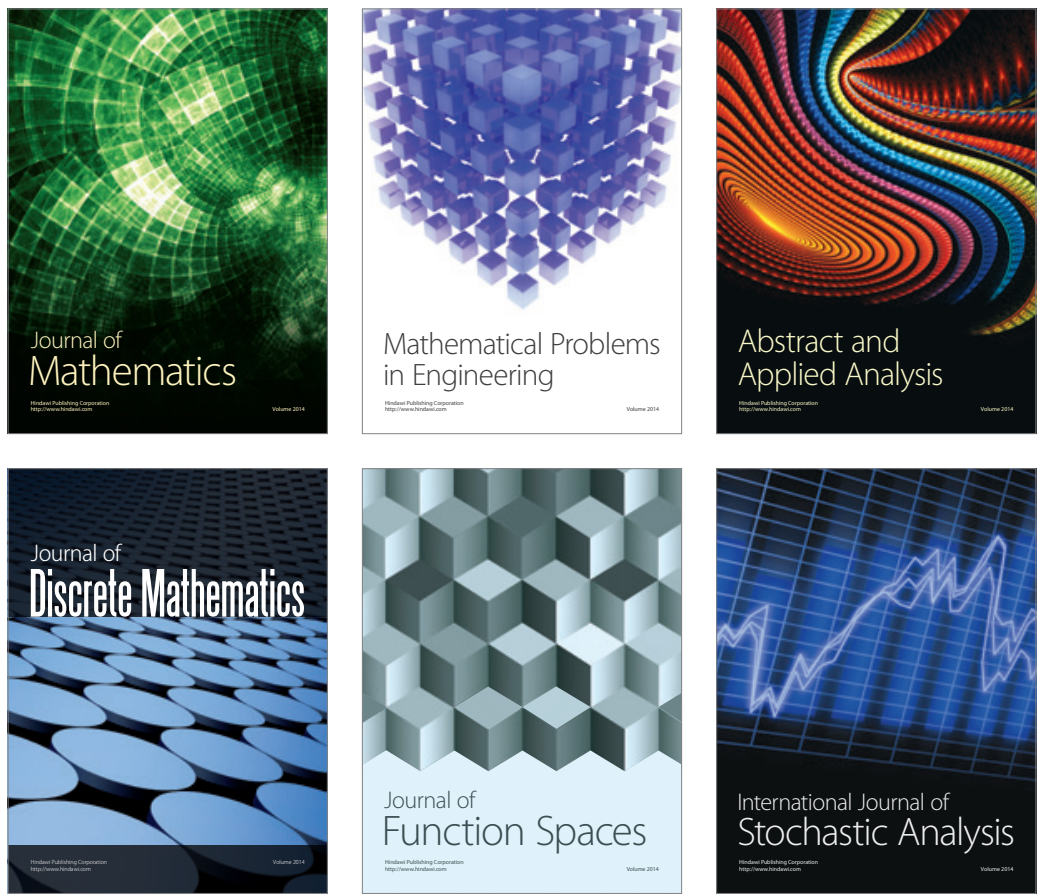

Journal of

Function Spaces

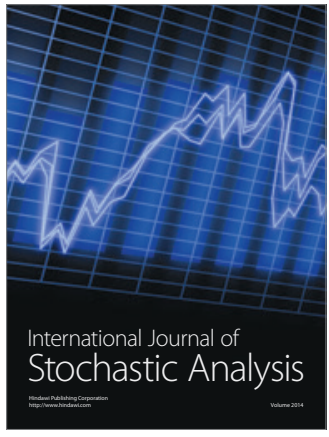

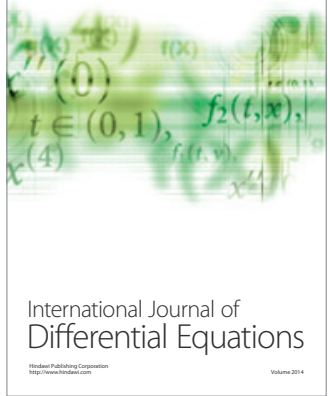
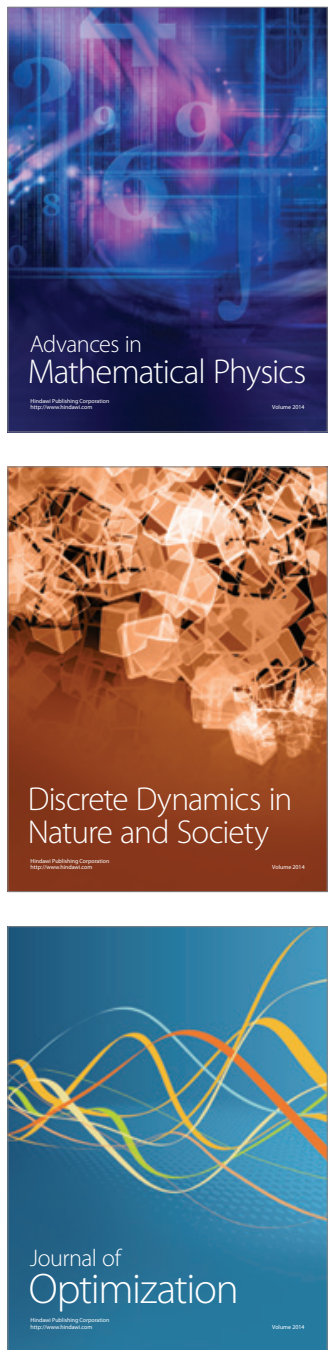